\title{
Preface
}

The GeoBigData'15 Workshop (2 October 2015), was organized by the ISPRS working group III/5 as an event of the ISPRS Geospatial Week 2015 (La Grande Motte, France, 28 September - 2 October 2015), and gathered researchers interested in the geospatial big data challenges, including storage, data management, processing, and rendering.

The GeoBigData'15 Workshop received a total of 23 submissions from various countries in Europe, USA, North Africa, and Australia, as 6 full papers and 17 extended abstracts. After a double blind review process, 3 full papers were accepted and $\mathbf{1 5}$ submissions were accepted as extended abstracts. Each paper received an average of three reviews from members of the Technical Papers Committee. The selected full papers and extended abstracts appeared respectively in issues of the ISPRS Annals and Archives of the Photogrammetry, Remote Sensing and Spatial Information Sciences. Selected research papers mainly address the following topics: extensions of big data frameworks to deal with geospatial big data; processing and rendering of geospatial big data; challenges in geospatial big data storage, processing and rendering; geospatial big data tools, services, and infrastructures on clouds. Full papers were presented in an oral session and submissions accepted as extended abstracts were presented both as regular interactive posters during a joint session with the GeoVIS, CMRT and ISA events and as a special oral session consisting of flash 5 ' talks.

Finally, the GeoBigData'15 Workshop has hosted one keynote talk by Rob Emanuele (Azavea, USA) on "The State of Distributed Processing in Open Source Geospatial Software".

Full papers were presented in an oral session and submissions accepted as extended abstracts were presented both at an interactive poster session and a flash $5^{\prime}$ talk session.

The GeoBigData'15 Workshop has also included a special oral session for the IQmulus Processing Contest (IQPC'15), chaired by Marco Attene (CNR-IMATI, Italy) and Silvia Biasotti (CNR-IMATI, Italy), with three main tracks: (i) Evaluation of $2 D$ footprints automatically generated from urban LIDAR data, which was organized by Dr. Linh Truong-Hong, University College Dublin, Ireland - Prof. Debra Laefer, University College Dublin, Ireland; (ii) Water detection and classification on multi-source remote sensing and terrain data, which was organized by Daniel Kristof (Institute of Geodesy, Cartography and Remote Sensing "FOMI" Budapest, Hungary; and (iii) Individual tree extraction, which was organized by Ben Gorte, Delft University of Technology, The Netherlands.

The GeoBigData'15 Workshop Chairs wish to acknowledge the support of the FP7 Integrating Project IQmulus, FP7-ICT-2011-318787, "A High-volume Fusion and Analysis Platform for Geospatial Point Clouds, Coverages and Volumetric Data Sets", and thank the IQmulus Project for choosing GeoBigData'15 to be the event for the organization of the IQmulus second Workshop. We would also like to thank all of the members of the Technical Papers Committee for their work in terms of reviewing papers.

\section{CONFERENCE CHAIRS}

Mathieu Brédif, IGN (FR)

Guiseppe Patanè, CNR-IMATI (IT)

Tor Dokken, SINTEF (NO)

\author{
PROGRAM COMMITTEE \\ Marco Attene, CNR-IMATI (IT) \\ Jan Boehm, UCL (UK) \\ Bailin Deng, EPFL (CH) \\ Rob Emanuele, Azavea (USA) \\ Roderik Lindenbergh, TUDelft (NL) \\ Clément Mallet, IGN (FR) \\ Frank Michel, Fraunhofer IGD (DE) \\ Mohamed F. Mokbel, Univ. of Minnesota (USA) \\ Nicolas Paparoditis, IGN (FR) \\ Michela Spagnuolo, CNR-IMATI (IT) \\ André Stumpf, UBO (FR) \\ Bruno Vallet, IGN (FR)
}




\section{$\underline{\text { Vitae }}$}

Mathieu Brédif is a research scientist at the IGN/MATIS laboratory. He obtained his PhD from Telecom ParisTech, France (2010), after completing its graduate studies at the Ecole Polytechnique, France and Stanford University (2004). He is chairing the ISPRS III/5 working group on computer graphics and remote sensing. His research interests include 3D reconstruction techniques, image-based modeling and rendering, computational geometry, stochastic geometry, image processing, pattern recognition, inverse rendering and virtual reality.

Giuseppe Patanè is researcher at CNR-IMATI, Italian National Research Council, Institute for Applied Mathematics and Information Technologies, and member of the Shape Modeling Group. He received a Master (1999) and a Ph.D. (2005) in Mathematics from the University of Genova; in 2000, he was research fellow of the Italian Institute of Advance Mathematics "F. Severi", University of Milano. His main research interests include shape modeling and analysis, geometry processing, computer graphics, and numerical analysis.

Tor Dokken is Chief Scientist and Research Manager of the geometry group in the Department of Applied Mathematics in SINTEF ICT, Norway. He received a Master (1978) and a Dr. Philos degree (1997) in approximation theory and spline technology from the University of Oslo, Department of Informatics. He has worked in SINTEF since 1978 addressing challenges within Computer Aided Geometric Design. In the later years the focus has been Locally Refined Splines addressing challenges within Isogeometric Analysis and geometric bigdata. Dr. Dokken is the Coordinator of the IQmulus Integrating Project. 